\title{
HIGHLIGHTS
}

EPILEPSY

\section{A new therapeutic strategy in epileptic seizure suppression}

Manipulation of brain $\mathrm{pH}$ by inhalation of $5 \% \mathrm{CO}_{2}$ (standard carbogen) might offer a novel therapeutic strategy in the treatment of epilepsy, according to new research published in the journal Epilepsia.

The anticonvulsant effects of $\mathrm{CO}_{2}$ have long been known, but only highconcentration inhalations have previously been investigated in patients with

A portable carbogen application device. Adapted from an image provided by Prof. Kai Kaila.

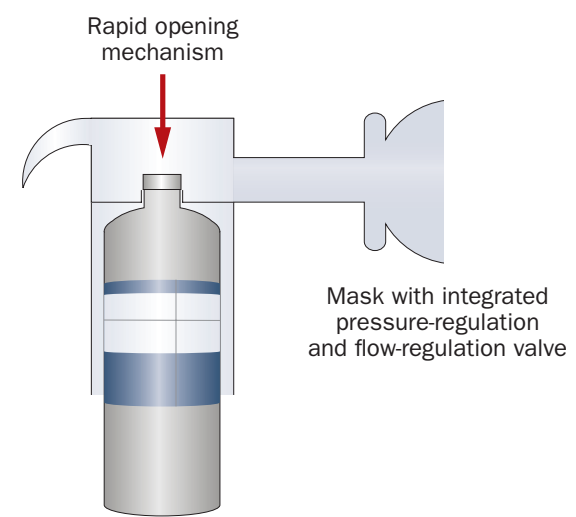

Replaceable gas container (for carbogen and air) epilepsy. "Activity-dependent acidosis is an important intrinsic factor in seizure termination, and breathing $5 \% \mathrm{CO}_{2}$ seems to selectively enhance this effect," explains neurobiologist and lead investigator Kai Kaila.

After inducing epileptic activity in rats and macaques, Tolner and co-workers observed that carbogen had "a surprisingly potent and fast anticonvulsant action, which provided a firm basis for the human study," comments Kaila.

The team went on to investigate the effects of carbogen after seizure generation in patients with epilepsy. Consistent with the animal studies, carbogen suppressed seizure activity in $<1-2$ min after administration.

In both the animal and human studies, the effects were examined in adults, but immature brains are known to be even more sensitive to $\mathrm{pH}$ changes. In another new paper published in Annals of Neurology, the same team report that in a rodent model of birth asphyxia, in which postasphyxic alkalosis is triggered by intense breathing, the seizures can be successfully suppressed through the use of carbogen.

The researchers hope that their work will pave the way for more-systematic developments and evaluations of $\mathrm{CO}_{2}$ therapy, and that in the future carbogen could be administered by paramedics or in the emergency room. Moreover, since some patients with chronic epilepsy can anticipate the start of a seizure, these new findings might provide a prophylactic treatment that can be administered, under professional supervision, in the home.

Among its advantages is the fact that carbogen is inexpensive, which, Kaila points out, "is an important point when considering clinical practices in developing countries."

Carolyn McSharry

Original article Tolner, E. A. et al. Five percent $\mathrm{CO}_{2}$ is a potent, fast-acting inhalation anticonvulsant. Epilepsia doi:10.1111/j.1528-1167.2010.02731.x

Further reading Helmy, M. M. et al. Brain alkalosis causes birth asphyxia seizures, suggesting therapeutic strategy. Ann. Neurol. doi:10.1002/ana.22223 